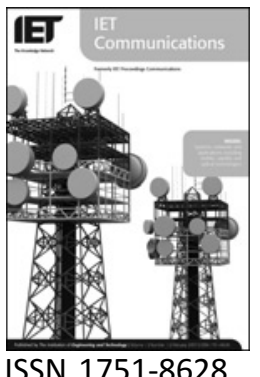

\title{
Spatial data stream multiplexing scheme for high-throughput WLANs
}

\section{A. Gravalos M. Hadjinicolaou Q. Ni R. Nilavalan}

Centre for Media and Communications Research, Brunel University, Uxbridge, Middlesex UB8 3PH, UK E-mail: Qiang.Ni@brunel.ac.uk

\begin{abstract}
A novel scheme using spatial data stream multiplexing (SDSM) in the upcoming multiple-input multipleoutput (MIMO)-based IEEE 802.11n physical layer is proposed. It is shown that with SDSM, the same data rate can be achieved by using less number of transmit and receive antennas and therefore this scheme can reduce the number of antennas which results in reducing mutual coupling effects, hardware costs and implementation complexities. The maximum data rates that can be achieved using a $2 \times 2 \mathrm{MIMO}$ system is $270 \mathrm{Mbps}$ and for a $4 \times 4$ MIMO system is 540 Mbps. The same data rates can be achieved using the SDSM technique which reduces the $2 \times 2 \mathrm{MIMO}$ system to $1 \times 1$ SISO system and the $4 \times 4 \mathrm{MIMO}$ system to a $2 \times 2 \mathrm{MIMO}$ system.
\end{abstract}

\section{Introduction}

Wireless is an attractive technology that offers great potentials in satisfying the increasing demand for low deployment cost and high-speed network connection. Throughput in wireless communication systems can be achieved either by increasing the number of antennas and hence the number of the spatial data streams or doubling the bandwidth of the channel. In the ideal case the maximum data rate achieved by the multiple-input multiple-output (MIMO) technique is $n_{\mathrm{t}} \times n_{\mathrm{r}}$ that of the data rate achieved by the SISO system $\left(n_{\mathrm{t}}\right.$ is the number of transmit antennas and $n_{\mathrm{r}}$ is the number of receive antennas).

Significant technological advancements are performed in the upcoming 802.11n standard in order to provide highthroughput WLANs $[1,2]$. A number of major proposals have been submitted by several company groups, for example, TGnSync [3], WWISE [4] and MITMOT [5]. Very recently these groups have agreed in submitting a unified proposal [6] to the IEEE 802.11n task group. The proposed 802.11n is based on MIMO antenna technology and orthogonal frequency division multiplexing (OFDM) modulation with advances in error correction and power efficiency.

OFDM is a multi-carrier transmission technique employed to reduce the intersymbol interference (ISI) effect of wireless systems with small symbol periods. As a result, the capacity in wireless systems is improved. Another promising transmission technique is MIMO antennas which enables the enhancement of capacity and performance with low bit-error-rate (BER) proportional to the number of antennas. Therefore high spectrally efficient wireless transmissions can be produced by using multiple transmit and receive antennas. The data rates of next-generation wireless communication systems using frequency selective fading environments can be improved through the combination of MIMO-OFDM signal processing.

The idea of MIMO systems is that, with a given arbitrary wireless communication system, there is a link where the transmitting and receiving ends are equipped with multiple antenna elements [7]. The signals produced by both types of antennas combine in such a way, which results in a reduced $\mathrm{BER}$ or increased data rates (bits/sec). A major feature of a MIMO system is its ability to utilise multipath propagation to increase the transmission efficiency and channel capacity by the use of random fading and multipath delay spread. In this way many orders of magnitude enhancement can be achieved in wireless communications without the use of extra spectrum [7]. The reliability of wireless communications is the product of diversity techniques such as temporal diversity, frequency diversity or antenna diversity [8].

In order to achieve the full diversity promised by transmitting and receiving antennas, space-time block code 
(STBC) is used [9]. The employment of more than one antenna at the transmitter and the proper coding of data across the transmitting antennas physical layers ( $\mathrm{PHY}$ ) with STBC guarantee an increased data rate with minimal decoding complexity at the receiver [10]. STBC technique is a special form of PHY diversity, which is a complex combination of coding theory, matrix algebra and signal processing [10]. It is a technique that operates on a block of input symbols producing a matrix and outputs whose columns and rows represent time and antennas, respectively. Therefore STBCs can be effectively used to exploit the advantage of MIMO systems. With STBCs, the channel state information (CSI) is suggested to be available at the receiver and is practically calculated by the use of training symbols and pilot tones.

While the combination of MIMO-OFDM with STBC techniques can increase the throughput, at the mobile terminal side the space is limited. In [11], it is shown that the capacity of MIMO system is reduced when the signals at the receiver are correlated. Therefore closely spaced antennas correlate the channel fading. As a result the capacity is reduced when the antenna spacing $d$ is less than $\lambda / 2$, where $\lambda$ is the carrier wavelength [12]. Mutual coupling between closely spaced antenna elements is also a potential problem. The mutual coupling between closely spaced antennas can modify antenna radiation patterns [13] resulting in possible degradation of the system performance.

Mutual coupling is one of the most important parameters affecting MIMO performance [14-18]. This effect takes place due to the close spacing of antennas in a MIMO configuration. Studies performed on mutual coupling and antenna termination on the capacity [19-21] had led to some important conclusions. First that the capacity obtained from two coupled dipoles is higher than that of uncoupled antennas for small dipole spacing due to the induced angle diversity combined with power collection capability of coupled antennas [19, 20]. However, for a fixed-length array, the strong coupling between elements packed into the same space will lead to an upper bound on capacity performance where a significant capacity reduction is observed compared with a system where coupling is neglected for spacing smaller than $\lambda / 2$ [22].

Recently, some works have been reported on the design and performance analysis for space-time block coded MIMO-OFDM wireless communication systems. For the two transmit antennas, a 'full-diversity full-rate' diversity only strategy has been proposed [23]. However, for the general case of the transmit antennas larger than two, if a diversity-only design is adopted, only STBC rate equalling to $1 / 2$ full diversity exists, although STBC rate $3 / 4$ exists for the three and four transmit antennas [6]. It has also been shown that STBC adaptivity in a $2 \times 2$ antennas MIMO-OFDM system can bring a small advantage over an unloaded system operating at the same rate in the absence of outer coding [7].
Although there is extensive work carried out in MIMOOFDM system design [24], little work has been reported in the literature regarding to the reduction of transmit/ receive antennas. This paper proposes a novel technique called spatial data stream multiplexing (SDSM). The goal of this technique is to minimise the number of antennas and as a result reduce mutual coupling effects, hardware costs and implementation complexities while maintaining the high throughput.

In this paper, the number of symbols for each spatial stream is set to 16 (an average payload data) with $5 / 6$ forward error correction (FEC) and 64-QAM modulation technique. Therefore the use of the buffer at the transmitter (SDSM) and receiver (SDSD) sides, respectively, accomplishes the reduction of the number of the antennas. Hence, this technique reduces mutual coupling effects, hardware costs and implementation complexities. The effects of mutual coupling on antenna patterns are obvious from the shape of the radiation patterns (as shown in Figs. 5 and 6). The number of antennas by this technique is reduced by the inclusion of a mathematic operation in the design of the PHY layer. Therefore the data streams are multiplexed in time. As a result, the mutual coupling effect is decreased while the bandwidth is increased.

More particularly the performance of the complete SDSM MIMO-OFDM employing Rayleigh flat fading channel is evaluated whose processing is implemented in systems with two and four transmit and receive antennas, respectively, with 64-QAM modulation and 5/6 forward error correction code using STBC code rate 1 . The simulation results obtained are compared with those from a conventional MIMO-OFDM $2 \times 2$ antenna system. Furthermore, comparisons were also made under different source powers using near field plots to estimate the coupling effects.

The outline of this paper is organised as follows. In Section 2 , the description of the system model implemented is introduced. In Section 3, the numerical results obtained from the simulations are presented. Finally, Section 4 contains the conclusions drawn from our work.

\section{Design and analysis of SDSM model}

\subsection{Idea of SDSM}

SDSM and spatial data stream demultiplexing (SDSD) were incorporated in the system design as parallel-to-serial and serial-to-parallel conversion of data streams for the transmitter and the receiver, respectively. The mathematical model of the considered SDSM system will be presented in Section 2.2. In order to achieve high data rates, MIMO antennas technologies were proposed in the 802.11n standard draft. However in practice, the number of antennas is constrained by the size of the devices. According to 
recent studies [22] the minimum distance between antenna elements so as to avoid the mutual coupling effect is $\lambda / 2$. This distance limitation generates the limitation of the number of antennas that can be used in a system. Therefore in an effort to increase the number of antennas while maintaining the desired space between antenna elements has as a result the creation of the SDSM and SDSD technique. Hence this scheme can achieve the desired highthroughput data rates or possibly even higher rates in future model designs by restricting the mutual coupling effect and system size.

The novelty behind SDSM technique is to reduce the number of antennas in a MIMO-OFDM transmitter/ receiver configuration. This results in a very useful system for handheld/mobile devices while achieving the same throughput in a higher order system. Although there is a trade-off between bandwidth and reduction of antennas, the practical advantages are significant in terms of the size limitations of mobile devices in some applications.

SDSM, at the transmitter, and SDSD, at the receiver, blocks function as buffers. They operate as storage medium for collecting digitally encoded coherent signals from each spatial stream into concatenated groups that are transmitted as a unit. The size of the buffer depends on the data size of the transmitted signal where the data size depends on the FEC and the modulation technique used for each data rate.

\subsection{Model description}

In 802.11n PHY layer, at the transmitter side a convolutional encoder is chosen to encode the binary source data, see Fig. 1. An encoder parser is a serial-to-parallel block that divides the data stream feed from source. The encoder stream, the number of encoder streams is denoted by $N_{\mathrm{ES}}$, comprises convolutional encoder, puncturer, interleaver and a modulator whose input is binary random data. A puncturer block has been added in order to achieve the code rates before the interleaver. The interleaver block is fed with coded bits from the convolutional encoder prior to a rectangular QAM baseband modulator using the Grey code for mapping the symbols. The stream parser module divides the output of the encoded stream into spatial streams $\left(N_{\mathrm{SS}}\right)$ by assigning each frame for feeding the OFDM multiplexing and then the inverse fast Fourier transform (IFFT) process converts the frequency symbols into the time domain to the respective transmit antenna. A cyclic prefix has been added to avoid the ISI interference. The SDSM block has been added in order to reduce the number of the antennas, which in turn minimises the mutual coupling effects, hardware costs and implementation complexities. Using the STBC code, the data passes to the receiver through Rayleigh flat fading channel using $4 \times 4$ antennas. The process of the transmitted signal at the receiver passes via the next blocks in order to receive the appropriate data. Hence, the output complex baseband signal $x_{i}^{k}(t)$ is filtered and converted to a radio frequency $(\mathrm{RF})$ signal with a carrier frequency $f_{\mathrm{c}}^{k}[4]$.

$$
x_{i}^{k}(t)=\sqrt{\frac{E}{N_{\mathrm{T} x}}} \sum_{n=0}^{N-1} d_{i}^{k}(n) \cdot \exp \left\{j \omega\left(t-T_{\mathrm{GI}}\right)\right\}
$$

for $i=1,2, \ldots, N_{\mathrm{T} x}$ transmit antennas and $k=1,2 \ldots, K$ OFDM blocks, where $E$ is the average energy per transmitted symbol which is independent of the number of transmit antennas. $d_{i}^{k}(n)$ is the complex signal that is transmitted through the $n$th subcarrier via the $i$ th transmit antenna during the $k$ th OFDM period. $\omega=2 \pi n \Delta_{f}$, and $\Delta_{f}=1 /\left(N T_{\mathrm{s}}\right)$ is the frequency separation between two adjacent subcarriers. $T_{\mathrm{GI}}$ is the guard interval duration

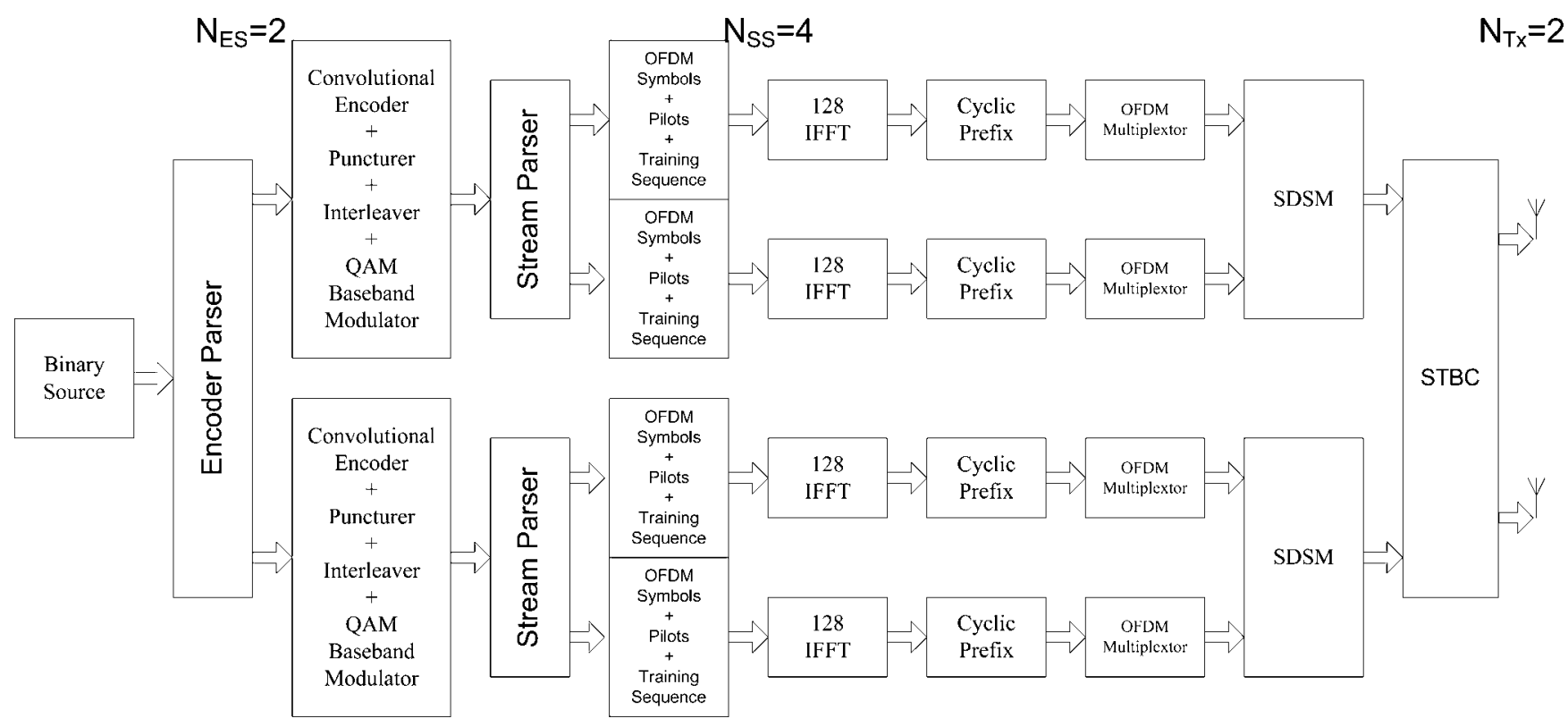

Figure 1 Transmitter MIMO-OFDM PHY layer model with SDSM 
$T_{\mathrm{GI}}=N T_{\mathrm{s}} / 4$ [25]. Therefore the signal operates in the interval[ $\left.T_{\mathrm{GI}}, T_{\mathrm{GI}}+N T_{\mathrm{s}}\right]$.

This signal contains the multiplexed $z$ th data streams (SDSM) of $k$ th OFDM block propagated from the $i$ th transmit antenna. Therefore the transmitted multiplexed OFDM signal at the $i$ th transmit antenna can be related to the complex baseband signal by

$s_{i}(t)=\sum_{z=0}^{Z-1} \sum_{k=0}^{K-1} \operatorname{Re}\left\{x_{i}^{k}\left(t-k T_{\mathrm{SYM}}\right) \cdot \exp \left(j 2 \pi(1+z) f_{\mathrm{c}}^{k} t\right)\right\}$

where, $\operatorname{Re}\{$.$\} represents the real part of the complex$ argument and $T_{\mathrm{SYM}}=T_{\mathrm{GI}}+T_{\mathrm{FFT}}+T_{\mathrm{GI} 2}$ where $T_{\mathrm{GI} 2}$ represents the training symbol guard duration. Hence, the actual RF signal over the air is the double sum of RF multiplexed signals emanated from each transmit antenna simultaneously.

On the receiver PHY layer system model, Fig. 2, the spatial data streams from each received antenna is demultiplexed (SDSD). Then the OFDM frames are demultiplexed. The cyclic prefix that has been added to the transmitter is now removed. The FFT reconverts the process to the original state from time domain to the frequency domain. The training and pilot synchronisation signals are removed whereas a frequency domain equaliser and a minimum mean square (MMSE) channel estimator have been added. The data passes through the demodulator, deinterleaver, unpuncturer and Viterbi decoder. The decoder stream, the number of decoder stream is denoted by $N_{\mathrm{DS}}$, combines the decoded bits and the information data reach the sink.

Therefore the received signals $r_{j}^{k}(n)$ at the $n$th subcarrier and the $j$ th receive antenna during the $k$ th OFDM symbol duration is expressed as

$$
r_{j}^{k}(n)=\sqrt{\frac{E}{N_{\mathrm{T} x}}} \sum_{n=0}^{N_{\mathrm{T} x}-1} H_{i j}^{k}(n) \cdot d_{i}^{k}(n)+w_{j}^{k}(n)
$$

where $w_{j}^{k}(n)$ is the additive white Gaussian noise. $H_{i j}^{k}(n)$ is the frequency response of the channel

$$
H_{i j}^{k}(n)=\sum_{l=0}^{L-1} a_{i j}^{k}(l) \cdot \exp \left\{-j n 2 \pi f \tau_{l}\right\}
$$

and where $a_{i j}^{k}(l)$ is the multipath gain coefficient for $L$ available paths and $\tau_{l}$ is the path delay of the $l$ th path.

As proposed in the 802.11n draft [4], the bandwidth of the OFDM signal is $40 \mathrm{MHz}$. In this case, the number of samples $(N \mathrm{~s})$ is 128 for an OFDM symbol which are equal to the number of subcarriers. The information-carrying data are encoded in the following modulation procedure. The binary source data sequence is first scrambled and then convolutionally encoded by the industrial standard rate $1 / 2$ encoder. The encoded output is then punctured according to the data rate requirement and is segmented into blocks of length $N_{\text {CBPS }}$, number of coded bits per symbol [4], each of which corresponds to an OFDM symbol. The binary data in each block is first interleaved among the subcarriers and then the information sequence is mapped in groups of bits into QAM symbols, which are used to modulate the different data carrying subcarriers. Each OFDM symbol in the OFDM data field employs $N$ s $=128$ subcarriers, 108 of which are used for data symbols and four for pilot symbols and one DC (Direct Current). There are also 15 null subcarriers with one in the centre and the other 14 on the two ends of the frequency band. The OFDM symbols, each of which consists of $N \mathrm{~s}=128$ samples, obtained via taking the IFFT of the data symbols, pilot symbols and nulls on



Figure 2 Receiver MIMO-OFDM PHY layer model with SDSD 
Table 1 Physical layer parameters

\begin{tabular}{|l|c|c|c|c|c|c|c|}
\hline $\begin{array}{c}\text { Data } \\
\text { rate, } \\
\text { Mbps }\end{array}$ & Modulation & $\begin{array}{c}\text { Code } \\
\text { rate }(R)\end{array}$ & $\begin{array}{c}\text { Number of data } \\
\text { subcarriers }\left(N_{\mathrm{SD}}\right)\end{array}$ & $\begin{array}{c}\text { Number of } \\
\text { pilots }\left(N_{\mathrm{SP}}\right)\end{array}$ & $\begin{array}{c}\text { Coded bits/ } \\
\text { MIMO-OFDM } \\
\text { symbol }\left(N_{\mathrm{CBPS}}\right)\end{array}$ & $\begin{array}{c}\text { Data bits/MIMO- } \\
\text { OFDM symbol } \\
\left(N_{\text {DBPS }}\right)\end{array}$ & $\begin{array}{c}\text { Packet } \\
\text { length, } \\
\text { bytes }\end{array}$ \\
\hline 270 & 64-QAM & $5 / 6$ & 6 & 108 & 4 & 1296 & 1080 \\
\hline 540 & 64-QAM & $5 / 6$ & 6 & 108 & 4 & 2592 & 2160 \\
\hline
\end{tabular}

these $N_{\mathrm{s}}$ subcarriers. To eliminate the ISI, each OFDM symbol is preceded by a guard interval, which contains the last samples of the OFDM symbol [4].

\subsection{Antenna design}

Although any type of antenna can be used for such systems an omni-directional dipole antenna has been proven to be widely used [26]. According to the IEEE 802.11n specifications the operation band is set to $2.4 / 5 \mathrm{GHz}$. Therefore the calculated wavelength is $\lambda=0.123 \mathrm{~m}$. The proposed antenna has the characteristic impedance of $50 \Omega$ which is a global standard and is fed by a short strip line representing the impedance source. The antenna source is located at the centre of the dipole.

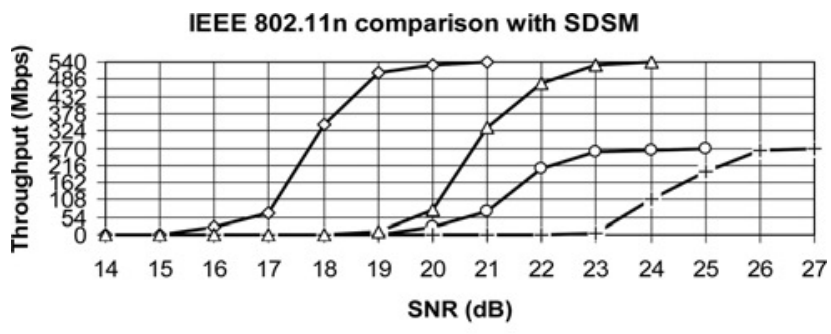

\begin{tabular}{|c|c|}
\hline$-\infty 270$ Mbps $2 \times 2$ system & $-+-270 \mathrm{Mbps}$ with SDSM (1x1) \\
\hline$\prec-540$ Mbps $4 \times 4$ system & $-\triangle 540$ Mbps with SDSM $(2 \times 2)$ \\
\hline
\end{tabular}

Figure 3 Throughput simulation results under Rayleigh flat fading channel

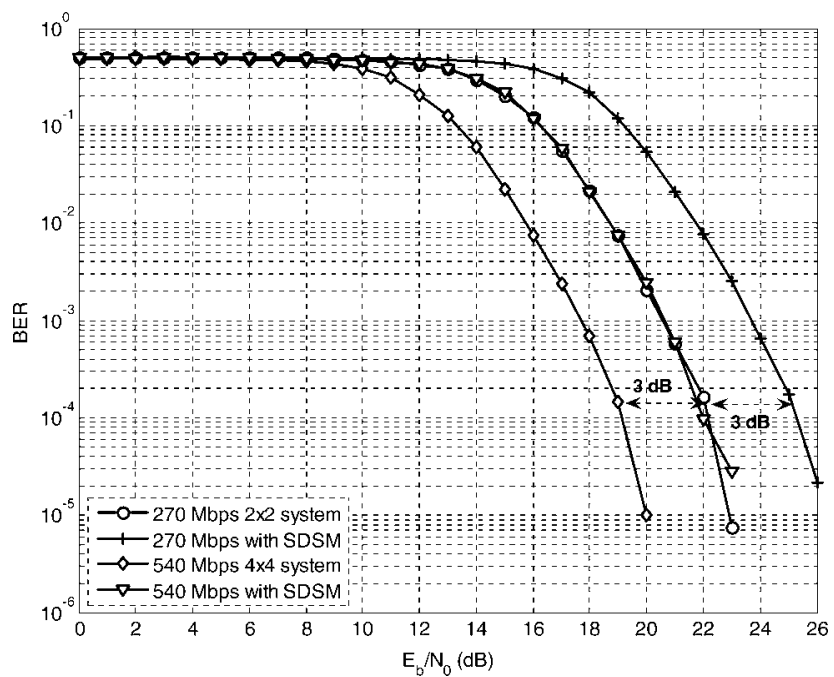

Figure 4 BER simulation results under Rayleigh flat fading channel
The main structure of the designed antenna has a length of $\lambda / 2=0.0615 \mathrm{~m}$ and radius $0.737 \mathrm{~mm}$ at $50 \Omega$ impedance. In addition, the resistive losses associated with the antenna were set to zero so as to maximise the efficiency of the

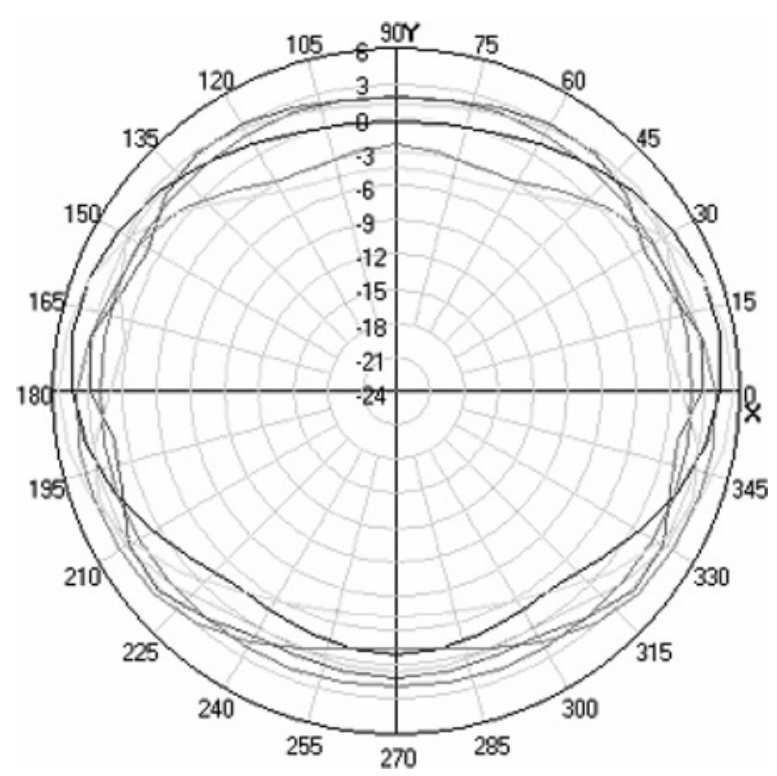

Figure 5 Horizontal plane polarisation of an omnidirectional dipole antenna

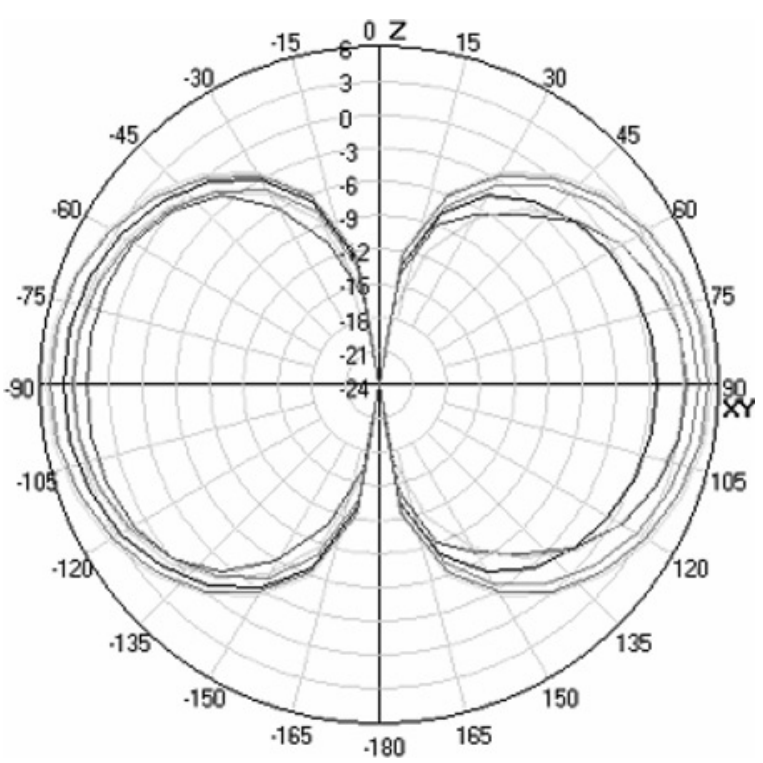

Figure 6 Vertical plane polarisation of an omni-directional dipole antenna 
Table 2 Legend for Figs. 5 and 6

\begin{tabular}{|l|c|c|c|c|c|c|}
\hline \multicolumn{1}{|c|}{ Colour } & Red & Black & Green & Blue & Purple & Cyan \\
\hline $\begin{array}{l}\text { number of } \\
\text { antennas }\end{array}$ & 1 & 2 & 2 & 2 & 4 & 4 \\
\hline dipole antennas & $\begin{array}{c}\text { reference } \\
\text { antenna }\end{array}$ & & & & $\begin{array}{c}\text { source at peak } \\
\text { antennas }\end{array}$ & $\begin{array}{c}\text { source at middle } \\
\text { antennas }\end{array}$ \\
\hline antenna spacing & $d=0$ & $d=\lambda / 2$ & $d=\lambda$ & $d=3 \lambda / 2$ & $d=\lambda / 2$ & $d=\lambda / 2$ \\
\hline
\end{tabular}

antenna. An input power of $1 \mathrm{~W}$ was considered for this analysis. In order to determine the input impedance and relate the load impedance to the reflection coefficient, $\Gamma$, the standing wave ratio (SWR) and Smith charts were used. The ratio of the maximum to minimum values of peak voltage amplitude measured along a standing wave is defined as the SWR.

$$
\mathrm{SWR}=\frac{1+|\Gamma|}{1-|\Gamma|}
$$

For the characteristic impedance of $50 \Omega$ the SWR is calculated as $1: 1.57$ by the numerical electromagnetic code (NEC) program. It is known that the efficiency of the antenna design is optimal when the SWR is 1:1. Furthermore, array configurations with multiple elements based on above antenna design and different inter element spacings were analysed. The radiation patterns and the coupling effects with different source powers for inter element spacings of $\lambda / 2, \lambda$ and $3 \lambda / 2$ were computed using the NEC software. The coupling effects and radiation patterns for the above spacings for $2 \times 2$ and $4 \times 4$ systems are discussed and presented below.

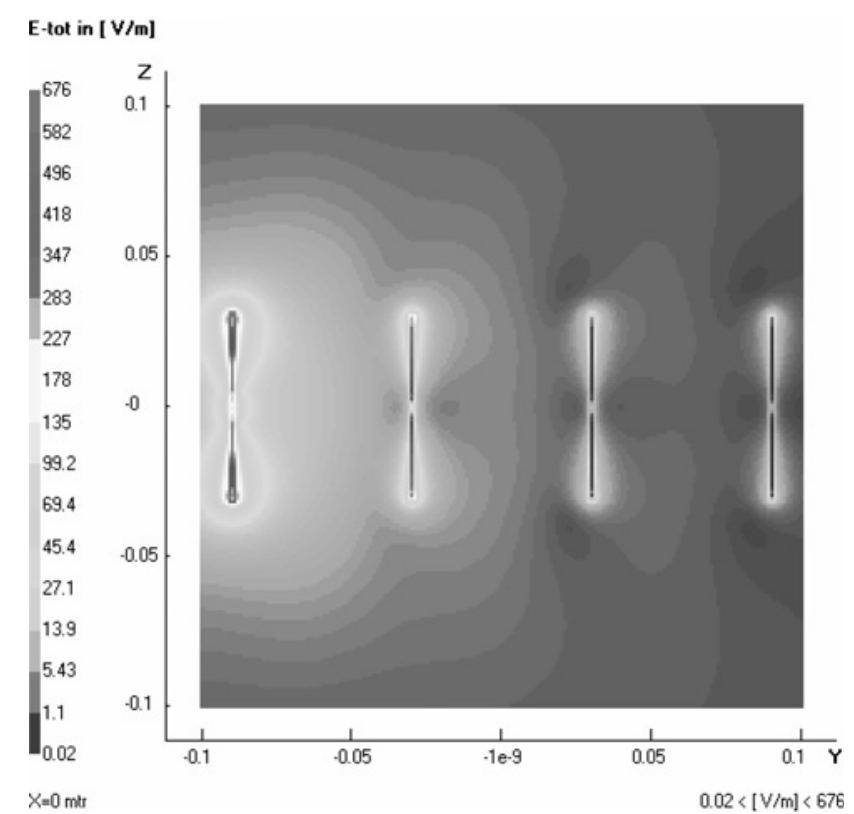

Figure 7 Near-field plot for $4 \times 4$ system with $1 W$ and $\lambda / 2$ separation

\section{Simulation results}

The scope of the simulation is to compare the original performance of the 802.11n with the new SDSM scheme. The highest data rates for each system have been selected according to the specifications [4]. The simulation experiments were carried in the MatLab platform (Table 1). These are: $270 \mathrm{Mbps}$ for a $2 \times 2 \mathrm{MIMO}$ system and $540 \mathrm{Mbps}$ for the $4 \times 4 \mathrm{MIMO}$ system according to the original design of $802.11 \mathrm{n}$. In this paper, we have shown that the same data rates can be achieved with less number of antennas using the SDSM technique. All the simulation pairs use a 5/6 forward error correction code rate with 64QAM modulation and STBC under Rayleigh flat fading channel. Therefore the number of antennas in each of the above systems is reduced to half, the data rate remains the same and the bandwidth of the channel is doubled to $80 \mathrm{MHz}$.

Figs. 3 and 4 show the performance of the four systems considered in this paper with respect to throughput and BER, respectively. From Fig. 4, it can be observed that a $2 \times 2$ MIMO system with SDSM achieves double data rates when compared with the standard $2 \times 2$ MIMO

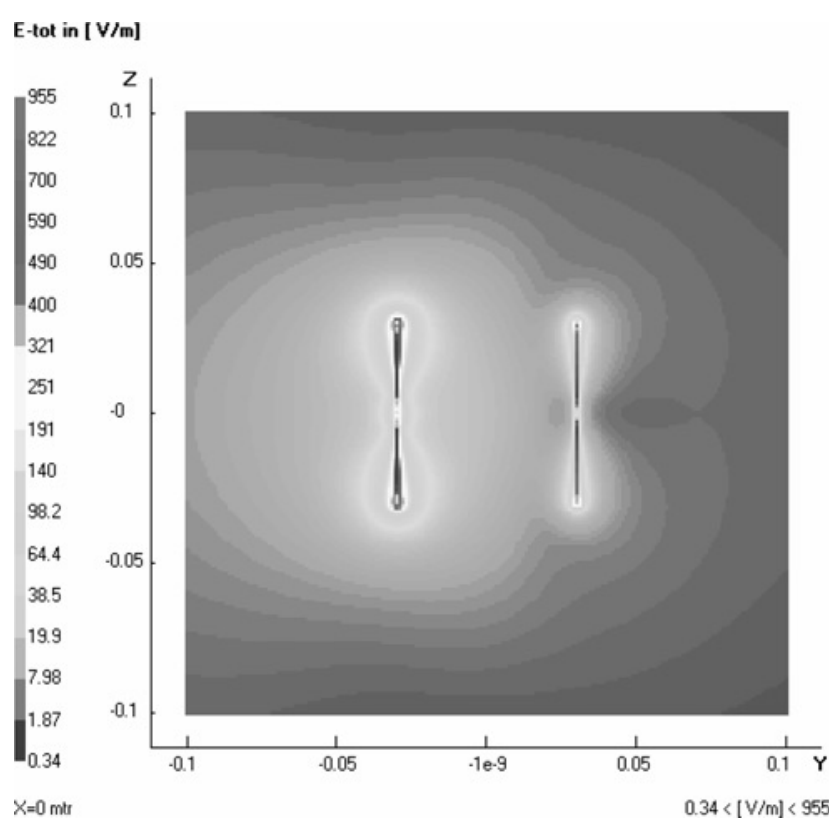

Figure 8 Near-field plot for $2 \times 2$ system with $2 W$ and $\lambda / 2$ separation 


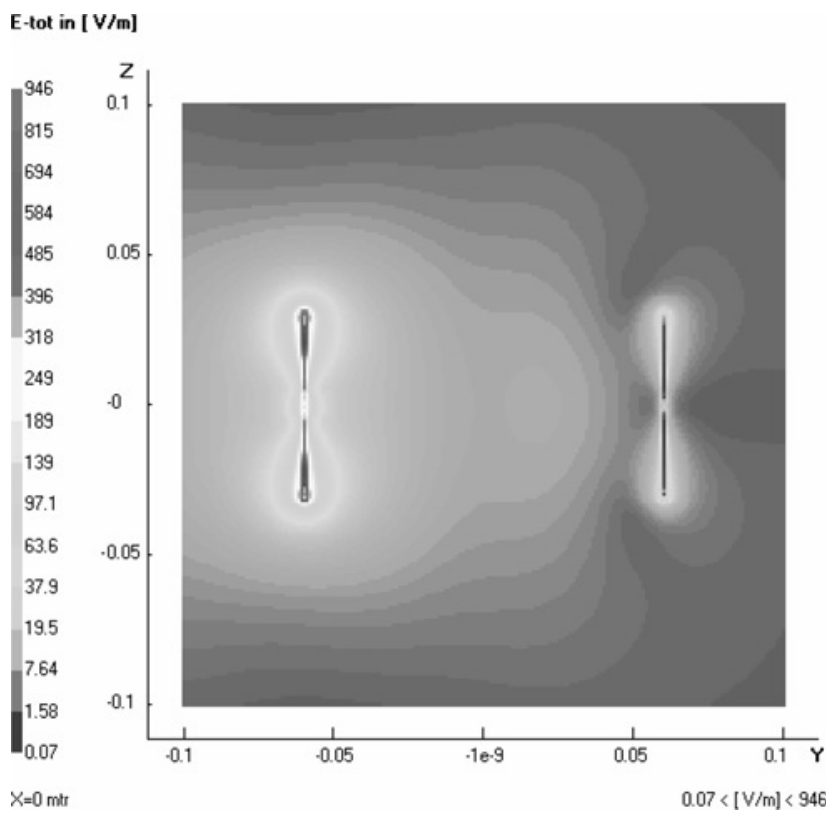

Figure 9 Near-field plot for $2 \times 2$ system with $2 W$ and $\lambda$ separation

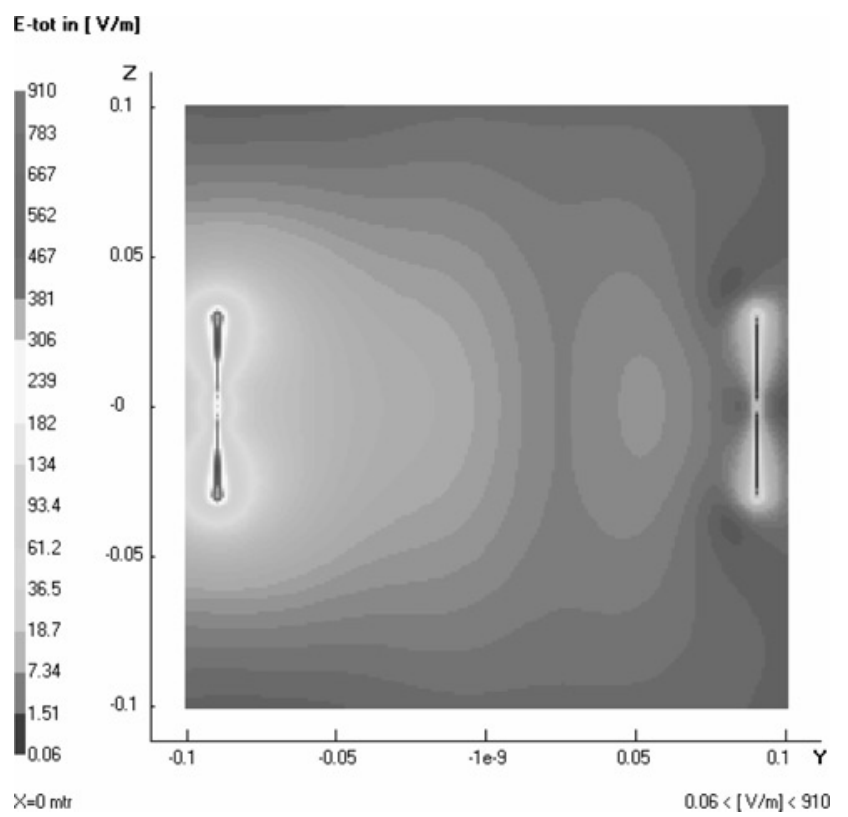

Figure 10 Near-field plot for $2 \times 2$ system with $2 W$ and $3 \lambda / 2$ separation system at the same SNR and BER. The same observation can be concluded for the $2 \times 2$ MIMO system with SDSM. Although Fig. 4 indicates that a standard $4 \times 4 \mathrm{MIMO}$ system requires less power $(3 \mathrm{~dB})$ than a $2 \times 2 \mathrm{MIMO}$ system with SDSM, the inclusion of the SDSM technique decreases the number of antennas. Also the cost of hardware implementation and the effects of mutual coupling are reduced significantly. In addition practical implementation issues can be simplified considerably as well.

Figs. 5 and 6 show the horizontal and vertical plane farfield radiation patterns for different array configuration where all elements are fed in phase. Table 2 summarises the number of elements and the inter element spacings considered in this NEC simulation. These results show the degradation suffered by multiple element configurations, especially the four element pattern is significantly degraded compared with the two element and single patterns in Fig. 5. Also, Fig. 6 shows some degradation for two element configuration with $\lambda / 2$ separation. Among the different configuration considered, only the two elements case with $3 \lambda / 2$ separation seems to give a radiation pattern similar in shape to a single dipole. These results clearly demonstrate the effects of mutual coupling on the radiation patterns and these effects will result in the degradation of the overall MIMO system performance. Reducing the number of elements will reduce the effects due to mutual coupling and hence provide a better performance.

Furthermore, Figs. 7-10 show the characteristics of the antenna elements in near field for $4 \times 4$ and $2 \times 2$ systems with different source powers where a single antenna was active and the rest were terminated with matched loads. These figures show the induced electric fields on neighbouring elements due to the currents on the active element. From Figs. 8-10, it can be clearly seen that the induced fields vary with antenna separation. Fig. 7 shows the coupling for a $4 \times 4$ systems with $1 \mathrm{~W}$ power which was analysed to compare the coupling effects with a $2 \times 2$ system with $2 \mathrm{~W}$ power. These induced fields due to mutual coupling will interfere with the received signals in practical systems and can also be estimated by the induced currents on the neighbouring elements. Table 3 shows the induced currents at the centre of the dipoles for these cases. These results clearly show that the induced current on the

Table 3 Induced currents on neighbouring elements

\begin{tabular}{|l|c|c|c|c|c|c|}
\hline \multirow{2}{*}{ System } & \multirow{2}{*}{ Power } & Separation & Element 1 & \multicolumn{3}{|c|}{ Induced currents, Amps } \\
\cline { 5 - 7 } & & & & Element 2 & Element 3 & Element 4 \\
\hline $4 \times 4$ & $1 \mathrm{~W}$ & $\lambda / 2$ & active & $2.376 \times 10^{-3}$ & $8.909 \times 10^{-4}$ & $5.707 \times 10^{-4}$ \\
\hline $2 \times 2$ & $2 \mathrm{~W}$ & $\lambda / 2$ & active & $3.365 \times 10^{-3}$ & & \\
\hline $2 \times 2$ & $2 \mathrm{~W}$ & $\lambda$ & active & $2.042 \times 10^{-3}$ & & \\
\hline $2 \times 2$ & $2 \mathrm{~W}$ & $3 \lambda / 2$ & active & $1.410 \times 10^{-3}$ & & \\
\hline
\end{tabular}


neighbouring element in a $2 \times 2$ system with separations in the range of $\lambda / 2-\lambda$ with twice the power is comparable with a $4 \times 4$ system with $\lambda / 2$ separation.

\section{Conclusion}

This paper has successfully demonstrated that the SDSM technique achieves a significant reduction in the number of transmit-receive antennas for MIMO-OFDM systems. Furthermore, the SDSM technique contributes in reducing the coupling effects between the antennas. Although there is a marginal increase in the hardware due to the addition of a parallel to serial converter at the transmitter and a serial to parallel converter at the receiver for implementing the SDSM technique the overall complexity of the system is cost-effective because of the significant reduction of the antennas. Simulation results have shown that MIMO systems with SDSM achieve double data rates when compared with standard MIMO systems at the same SNR and BER. This novel technique can offer higher data rates than the existing proposal by eliminating the present limitations for next generation WLAN systems. The SDSM technique offers the hardware benefit that more spatial streams can be used with less number of antennas and therefore higher data rates (>540 Mbps) can be achieved. Finally, we have shown that the overall performance of the 802.11n PHY layer MIMO-OFDMSDSM system has satisfied the requirements of the specification with respect to throughput at the higher data rate mode.

\section{Reference}

[1] WALdSChmidt C., KUhnert C., PAUli M., WiesbeCK W.: 'Handy MIMO', IEE Commun. Eng. Mag., 2005, 3, (1), pp. 22-25

[2] WALKO J.: 'Analysis - The ' $\mathrm{n}$ ' factor', IEE Commun. Eng. Mag., 2004-2005, 2, (6), p. 9

[3] TGn Sync proposal: doc.: Standard IEEE 802.11-04/ 0889r3

[4] Wwise proposal: High throughput extension to 802.11 n standard IEEE 802.11n draft 11-04-0886-00-000n

[5] Bonneville H., Jechoux B., Rollet R. et al.: 'Mac and mImo techniques for MOre throughput', IEEE 802.11n draft 11-04-1446r1, November 2004

[6] IEEE Draft amendment to standard: Enhancements for Higher Throughput, IEEE P802.11n /D1.0, March 2006

[7] Gebert D., ShAFI M., SHIOU D., SMITH P.J., NAGUib A.: 'From theory to practice: an overview of MIMO space-time coded wireless systems', IEEE J. Sel. Areas Commun., 2003, 21, (3), pp. 281-302
[8] TAROKH V., SESHADRI N., CALDERBANK A.R.: 'Space-time codes for high data rate wireless communications: performance criterion and code construction', IEEE Trans. Inf. Theory, 1998, 44, (2), pp. 744-765

[9] TAROKH V., JAFARKHANI H., CALDERBANK A.R.: 'Space-time block coding for wireless communications: performance results', IEEE J. Sel. Areas Commun.,, 1999, 17, (3), pp. 451-460

[10] STAMOULIS A., AL-DHAHIR N.: 'Impact of space-time block codes on 802.11 network throughput', IEEE Trans. Wirel. Commun., 2003, 2, (5), pp. 1029-1039

[11] FOSCHINI G.J., GANS M.J.: 'On limits of wireless communicaitons on fading environment when using multiple antennas', Wirel. Pers. Commun., 1998, 6, pp. 311-335

[12] JUNGNICKEL V., POHL V., VON HELMOT C.: 'Capacity of MIMO systems with closely spaced antennas', IEEE Commun. Lett., 2003, 7, (8), pp. 361-363

[13] TAGA T.: 'Characteristics of space-diversity branch using parallel dipole antennas in mobile radio communications', Electron. Commun. Jpn. 1, 1993, 76, (9), pp. 55-65

[14] ALLEN L.J., DIAMOND B.L.: 'Mutual coupling in array antennas', Technical Report, Lincoln Laboratory, M.I.T., Technical Report 424 (ESD-TR-66-443), 1966

[15] BALANIS C.A.: 'Antenna theory: analysis and design' (Wiley, New York, 1997)

[16] WALLACE J.W., JENSEN M.A.: 'Electromagnetic considerations for communicating on correlated MIMO channels with covariance information', IEEE Trans. Wirel. Commun., 2008, 7, (2), pp. 543-551

[17] LI F., ZHANG Q.T.: 'Transmission strategy for MIMO correlated Rayleigh fading channels with mutual coupling'. Proc. IEEE Int. Conf. Communications, ICC'07, Glasgow, UK, June 2007, pp. 1030-1035

[18] CHIU C.Y., CHENG C.H., MURCH R.D., ROWELL C.R.: 'Reduction of mutual coupling between closely-packed antenna elements', IEEE Trans. Antennas Propag, 2007, 55, (6), pp. $1732-1738$

[19] Wallace J.W., Jensen M.A.: 'The capacity of MIMO wireless systems with mutual coupling'. Proc. IEEE 56th Vehicular Technology Conf., Vancouver, British Columbia, Canada, 24-28 September 2002, vol. 2, pp. $696-700$

[20] wallace J.W., Jensen M.A.: 'Termination-dependent diversity performance of coupled antennas: Network theory analysis', IEEE Trans. Antennas Propag., 2004, 52, pp. 98-105 
[21] JANASWAMY R.: 'Effect of element mutual coupling on the capacity of fixed length linear arrays', IEEE Antennas Wirel. Propag. Lett., 2002, 1, (1), pp. 157-160

[22] JENSEN M.A., WALLACE J.W.: 'A review of antennas and propagation for MIMO wireless communications', IEEE Trans. Antennas Propag., 2004, 52, pp. $2810-2824$

[23] ALAMOUTI S.M.: 'A simple transmit diversity technique for wireless communications', IEEE J. Sel. Areas Commun., 1998, 16, (8), pp. 1451-1458
[24] STUBER G.L., BARRY J.R., MCLAUGHLIN S.W., LI Y., INGRAM M.A., PRATT T.G.: 'Broadband MIMO-OFDM wireless communications', Proc. IEEE, 2004, 92, pp. 271-294

[25] Gravalos A.G., hadjinicolaou M.G., NI Q.: 'Performance analysis of IEEE 802.11n under different STBC rates using 64-QAM'. IEEE Int. Symp. Wireless Pervasive Computing (ISWPC 07), San Juan, Puerto Rico, February 2007

[26] CHO Y.J., HWANG S.H., PARK S.O.: 'Printed antenna with folded non-uniform meander line for $2.4 / 5 \mathrm{GHz}$ WLAN bands', IEE Electron. Lett., 2005, 41, (14), pp. 786-788 